\title{
A content analysis in reverse logistics: a review
}

\begin{abstract}
The purpose of this paper is to provide a comprehensive review in the various publications on the concept of Reverse Logistics (RL) and the related areas within the period 1998-2012. The content analysis approach has been opted to collect the relevant information from different books, journals, and conferences. A broad review of literature in RL from its emergence until the recent discussions have been analyzed and compared in this research. The findings show that, the theoretical construct in RL has been initiated from the conjunction features in the waste management and logistics activities. This idea had been developed by introducing the new term as RL and its definitions and contents such as the activities; key drivers; barriers to use; material flow, and networks in RL. Furthermore, the findings present the various modelling in different aspects of RL, for instance, the mathematical modelling by applying the existence methods in Multi Attribute Decision-Making Models (MADM). In addition, the environmental concerns and governmental legislatives matters and impacts, which have been highlighted, recently, on RL have been deliberated. Hence, this paper would assist the researchers and practitioners to obtain a broad review of RL in the last decade and, also provide an agenda for the future researches.
\end{abstract}

Keyword: Reverse logistics; Review; Reverse logistics network; MADM 